\title{
Database Migration
}

\author{
Kanika Khetarpal \\ Department of Computer Science Engineering \\ Guru Tegh Bahadur Institute of Technology, New Delhi, Delhi, India \\ Neetu Singh \\ Department of Computer Science Engineering \\ Guru Tegh Bahadur Institute of Technology, New Delhi, Delhi, India
}

\begin{abstract}
Database Migrator is a tool which is used to migrate Database schema, primary keys and data from one source to another destination database. It validate the user credentials and verify them. Thus it is a very secure tool for database migration. It also has a graphical user interface which is very interactive. It doesn't allow system to install on but it can be operated from a different system for database migration. In other words it can be used on source machine or it can be operated from a remote machine which is not a part of source or destination system. Thus there is no need to install this tool on source machine which is more effective. It migrates data, database schema as well as the primary keys in the source database. Since it uses the credentials details provided by the user so it is very secure tool to migrate data from source database to destination database. It helps the individual user or a whole enterprise to migrate their whole database from one server to another very easily and effectively. After loading into the new system, results are subjected to data verification to determine whether data was accurately translated, is complete and supports processes in the new system. Data migration phases (design, extraction, cleansing, load, verification) for applications of machine to high complexity are commonly repeated several times before the new system is deployed.
\end{abstract}

\section{INTRODUCTION}

In present days the area of data migration is very topical. Various information systems and their databases are often transferred between different types of hardware or software equipment. This activity requires the proper coordination and management, because it is necessary transfer data and their structure to the new system correctly. Data migration is the process of moving data between storage units or entire computer systems. In order for this process to be efficient, powerful data extraction and data loading designs are critical. These designs help in mapping data, which is present on the current system, to the new system which is being implemented. One of the categories of data migration is database migration. Database migration is the process of moving the business logic, schema, physical data and database dependencies from a current system to a different/new system. Database migration is used when it is required to shift from one database vendor to another. This may be because of various reasons such as cost, capabilities, functionalities, requirements etc.

To achieve an effective data migration procedure, data on the old system is mapped to the new system utilizing a design for data extraction and data loading. The design relates old data formats to the new system's formats and requirements. Programmatic data migration may involve many phases but it minimally includes data extraction where data is read from the old system and data loading where data is written to the new system.

Now we would like to define the process that is used to migrate the database data.

The general process of data migration, which is used for database migration and data between different types of RDBMS is called ETL (Extract, transform and load) and consists of the following 3 steps:

1. Extracting data from the source database.

2. Transforming data into usable form for migration to the target database. 3. Migration of data to the target database.

The ETL process uses the terms source and target databases that are refined here:

- Source database - database of source RDBMS, from which data are migrated

- Target database - database of target RDBMS, into which data are migrated

There are a lot of reasons for data migration between various types of RDBMS, we would like to state here only few of them:

1. Upgrading to new version of the same RDBMS - in the case of regular upgrading of software equipment in the company, including new version of RDBMS, we need to migrate data to new RDBMS 
2. The existing RDBMS is insufficient - in the case of a large increase of stored data, insufficient speed or capacity of RDBMS

3. Change of company policy - in the case of changing security or another type of policy in company, we need to upgrade to better, more sophisticated RDBMS

4. Economical problems in company - this can lead to uninstalling commercial and expensive RDBMS and installing open-source and cheaper RDBMS instead

5. A lot of various applications and RDBMS - we need to unify various database and various RDBMS to one specific RDBMS to ensure better efficiency and consistency of data

6. Travelling of data- we need to travel data or share data between different numbers of systems.

\section{METHOD}

Database Migrator tool help in effective and systematic record keeping that is storing and retrieving of useful data. The database migrator tool is divided into four main modules.

1. Connection Provider module

2. Migrator module

3. Database Operation module

4. GUI module

Module 1:

\section{Connection Provider module:-}

1.

2. server.

3.

4.

5.

host servers.
It set up a secure connection to the database.

It connect two different databases on different Source request for a connection to destination. Destination approves the connection request.

A secure connection is established between two

\section{Module 2:}

Migrator module:-

1.

2.

3.

connection to the destination.
It fetches metadata from the source.

Data is fetched using metadata.

Data is transferred over the already established

\section{Module 3:}

\section{Database Operation module:-}

1.

2.

3.

connection to the destination.
Metadata is fetched from the source.

Data is fetched using metadata.

Data is transferred over the already established

\section{Module 4:}




\section{GUI module:-}

1.

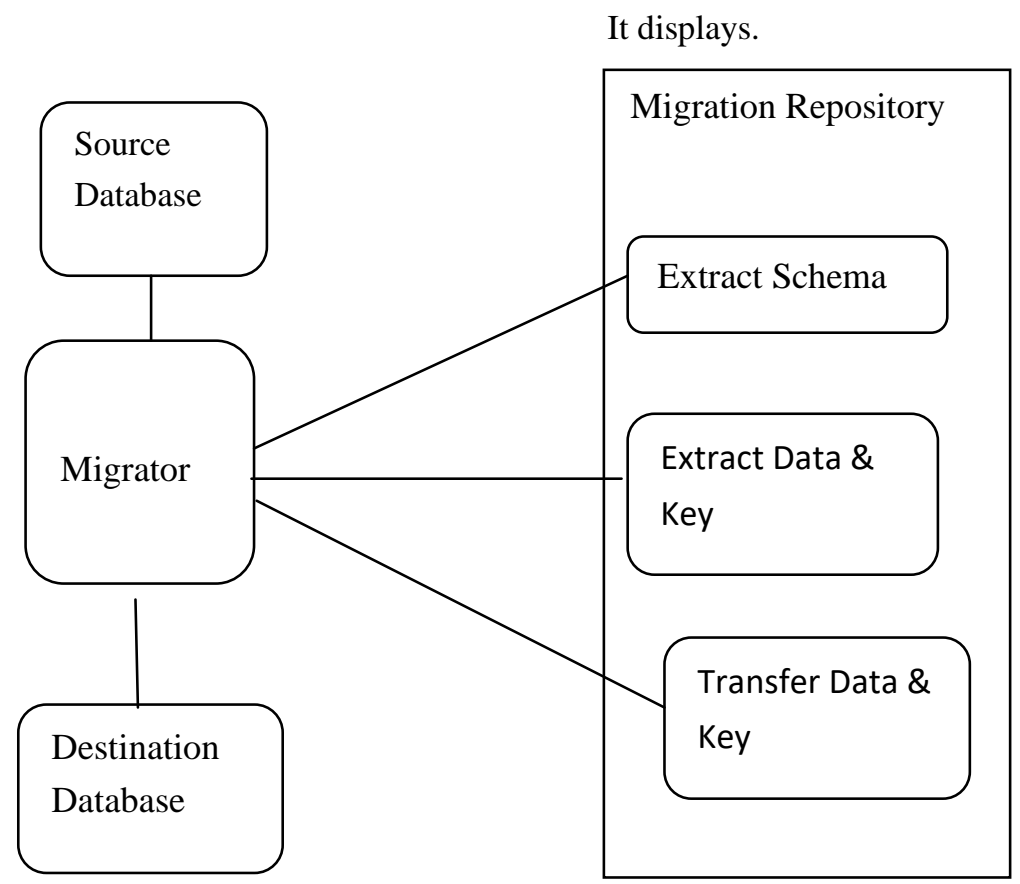

Figure 2.1 Basic Model of Database Migrator

In this figure the basic model of database migration is been shown. The migration repository is such that the migrator extract schema, extract data \& keys from the source database and transfer into the destination database Database migration generally result from the introduction of a new system. This may involve an application migration or consolidation in which one or more legacy systems are replaced or the deployment of an additional system that will sit alongside the existing applications. Whatever the specific nature of any data migration, the ultimate aim is to improve corporate performance and deliver competitive advantage.

Accurate data is the raw material that maximizes the value of enterprise applications. However, when existing data is migrated to the new target application, it can become apparent that it contains inaccuracies, unknown, and redundant and duplicate material. And although the data in the source system may be perfectly adequate for its current use, it may be wholly inadequate, in terms of content and structure, for the objective of the target system.

- Source Database

- Destination Database

- Source ip address and port number

- Destination ip address and port number

- Source database credentials

- Destination database credentials

\section{RESULTS}

Database Migrator is a tool which is used to migrate Database schema, primary keys and data from one source database to another destination database. It validate the user credentials and verify them. Thus it is very secure tool for database migration.

It also has a graphical user interface which is very interactive. It doesn't allow system to install on but it can be operated from different system for database migration. In other words it can be used on source machine or it can be operated from remote machine which is not part of source or destination system. Thus there is no need to install this tool on source machine which make it more effective.

It migrates data, database schema as well as primary keys in the source database. Since it uses the credentials details provided by the user so it is very secure tool to migrate data from source database to destination database. It helps the individual user or the whole enterprise to migrate their whole database from one server to another to another 
server effectively. After loading into the new system, results are subjected to data verification to determine whether data was accurately transmitted, is complete, and supports processes in the new system. During verification process their may be need for parallel run of both systems to identify areas of disparity and forestall erroneous data loss. Automated and manual data cleaning is commonly performed in migration to improve data quality, eliminate redundant data or obsolete information and match the requirements of the new system. Data migration phase (design, extraction, cleaning, load, verification) for applications of moderate to high complexity are commonly repeated several times before the new system is deployed.

\section{CONCLUSION}

Finally, we conclude that database migrator is actually one of the best data migrator tool by which we can migrate data easily and effectively. We can migrate data from source machine to the destination machine from a third remote machine.

It support MYSQL database server. It has very interactive graphical user interface. It is platform independent tool so can run the same program from any operating system without doing any changes.

It has a lot of features which make it better than other available tools for data migration but still there is lot to improve. For now this tool is only compatible with MYSQL database server but in future we will try to make it compatible with all the available database servers so that data can be migrated between different database servers.

\section{REFRENCES}

[1] Java 2 complete Reference ( $5^{\text {th }}$ edition).Author-Herbert Schildt , Published in 2005

[2] Thinking in java by Bruce Eckel Published in 1998

[3] The java Language Specification Author Gosling, james, joy, Bill, Published in 2004 http://docs.oracle.com/javase/specs/jls/se8/jls.pdf

[4] The java Virtual Machine Specification Author -Lindholm, Tim, Yellin, Frank, Published in 1999 http://docs.oracle.com/javase/specs/jvms/se8/jvms8.pdf

[5] Easy java Simulation Author-Esquembre, published in 2004

[6] Swings and Framing http://oopweb.com/Java/IntroToProgrammingUsingJava/VolumeFrames.html

[7] The integration of functions into logic programming: From theory to practice journal of Logic Programming, published in 1994, AuthorHanus M.

[8] Java Security Published in 1995 http://www-swiss.ai.mit.edu/jbank/javapaper/javapaper.html

[9] http://www.mysqltutorial.org/basic-mysql-tutorial.aspx/ 